\title{
A Study on the Chinese Research of Plato's Philosophy of Language
}

\author{
Shiqi Wang ${ }^{1 *}$ \\ ${ }^{1}$ Chengdu University of Technology, Chengdu, Sichuan,610000, China \\ *Corresponding author. Email:2960955867@qq.com
}

\begin{abstract}
Nowadays, in China, the research of Plato's language philosophy leaves a blank. Although Plato's language philosophy has been spread to China for a long time, scholars have translated many books of Plato and written books analyzing it. There are still limited scholars learning this subject, and few essays can be found on an academic website. Specifically, in CNKI, the number of articles on Plato's language philosophy was just one essay this year, and the highest amount was 4 in 2020. So, this article should firstly give a brief review of Plato's language philosophy, which are the theory of reference of names, the statement, and its nature, and the relationship between language and reality, and it aims to find out solutions of spreading Plato's language philosophy in China by adding some traditional and new-age Chinese philosophy elements into it, such as, Taoists and Marx and putting some properties of Plato's language philosophy into conventional Chinese philosophy, for example, the theory of name correction, for interpreting it and giving it more meanings. In this way, it is helpful to spread the language philosophy of Plato, encourage some research of it, and enrich the contents.
\end{abstract}

Keywords: language philosophy, Plato, Taoists, Marx, Confucius

\section{INTRODUCTION}

In modern philosophy, language philosophy plays a central role in this area, which aims at analyzing and interpreting human language. Through criticizing language, people can understand the relationship between the existence of themselves and the world. Moreover, Plato is thought to be the first philosopher researching western language philosophy. His work Kratylos is the first book discussing professional knowledge of the language and is the signals of the birth of western language philosophy[1]. In China, the values of Plato's language philosophy are giving some enlightenment views about emerging issues, enriching Chinese language philosophy and catching the era of linguistic steering.

However, although Plato was introduced into China in the early 20 years of 20th century and many scholars were translating his books, such as Yan Qun and Zhu Guangqian, the development of Plato's language philosophy is not wide enough. Few people think about spreading it in China by combining it with China's traditional language philosophy[2]. So, this article will find the number of articles on CNKI, and it aims at figuring out the solutions of disseminating it in China.
In general, the significance includes helping to spread Plato's language philosophy in China and improving its academic research, enriching the connotation of his language philosophy, giving more meanings of Chinese philosophy, encouraging the inspiration of learning it, and providing more solutions for spreading it.

\section{THE BRIEF STATEMENT OF PLATO'S LANGUAGE PHILOSOPHY}

\subsection{The Theory of Reference of Names}

Firstly, Plato disagreed with agreentism. Plato thought there were right or wrong statements, so as too the names, which made up the complete statements. Therefore, the names given by anyone could not be recognized as always right. Moreover, the objects must have some stable and perpetual essentials independent of people's minds. So, when naming the objects, people should use proper and natural tools to reflect the natural properties rather than arbitrarily referring to the names[3].

Secondly, Plato also could not accept naturalism. People could not understand the essentials of the objects, 
as the corporeal forms were unstable and metabolic, which resulted in people not naming any objects. For another, if the names were completely the same as the actual objects, there would be two objects and no one could distinguish the real ones[3].

In summary, if people utilized agreentism, the names could not be told from right to wrong. Furthermore, if people believed in naturalism, the objects could not be recognized from their names[3].

\subsection{The Statement and Its Nature}

Plato named statement as the combination of nouns and verbs, which provided information about now, past and future could teach people how to think and judge. Moreover factual statements related to existing objects, but false statements were things different from objective things[4].

For the emerging of false statements, Plato pointed out that the illusion in mind was a mix of feelings and judgments. In the situation of when people understood and felt the objects, logical judgment would be distorted. Then the statements would be true or false. When minds directed reflective shadows towards the objective imprints, the statements would be factual, while the minds disrupted the reflective shadows and directed them towards wrong imprints, then the judgment was false. Therefore, the only place false statements exist was the in conformity of feelings and minds[3].

However, Plato finally argued that people could not conclude that what someone knows is another thing he knows, as this argument already meant this person owned the knowledge. Nevertheless, in Plato's opinion, people could not figure out the nature of knowledge. If people concluded like this, the false statement was impossible. Therefore, he admitted that people should figure out the false statement rather than the unsuitability of minds and feelings[3].

\subsection{The Relationship Between Language and Reality}

Plato wrote any objects owned three things, and people should gain knowledge through these three things. Knowledge itself was the fourth thing and, the actual reality was the fifth. Therefore, there were: first, names; second, description; third, imagination; fourth, knowledge; fifth, actual reality of true objects. Moreover, logic had a close relationship with reality and the others were far away from it. Also, these four things owned the properties of inappropriateness and unsuitability, which implied themselves with specific shows by whatever the forms of language or figures, and these forms could be misguided by people's senses. Hence, no one could place the actual reality with logical thinking into the writing symbols expressions. In conclusion, Plato completely denied that language could reflect reality[5].

On the contrary, Plato did not object to language. When exploring the truth, Plato disagreed with directly learning them, like directly staring at the sun using eyes, but to use some indirect tools, for example, language, and he supposed that was the originals of all explorations [5].

On the whole, Plato's attitudes towards these theories were skeptical and negative. Although he did not clearly answer these questions, he put up with professional language philosophy directions and gave thinking of the quick solutions to these problems, which impacted on 'the linguistic turn'. For instance, Kripke accepted the opinion of agreentism, while Frege and Russell had clear thoughts about naturalism. Meanwhile, Plato's spirit of analysis means the process of researching beginning from the simplest things to the most complex objects, and this thought had inspired Wittgenstein's ideas of Logical Atomism and the principle of simplicity when exploring language philosophy.[6]

\section{THE COGNITIVE DEGREE OF PLATO'S LANGUAGE PHILOSOPHY}

Below are two graphs based on www.cnki.com, show the number of essays about Plato's language philosophy.

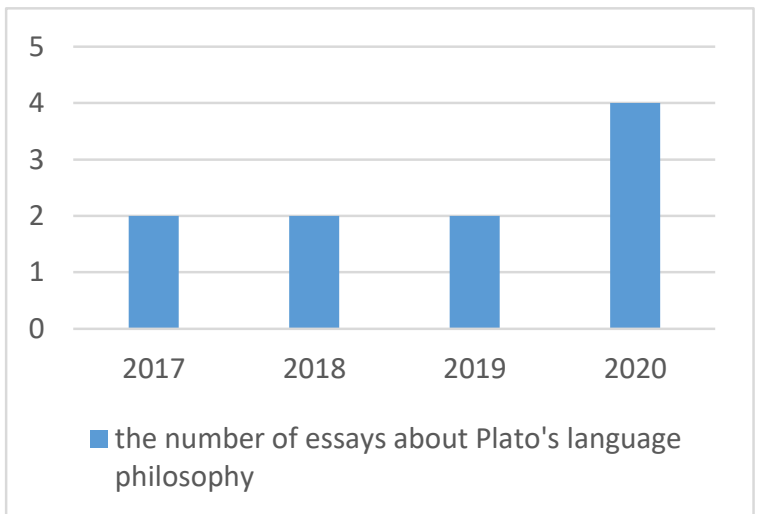

Figure 1. The number of essays about Plato's language philosophy

The graph illustrates that there were eleven essays about Plato's language philosophy in the recent five years and for the single year, the amount could not go beyond four. In 2020, the number reached the highest level, which was only four. Moreover, for 2021, the number declined $75 \%$ and was just one essay published. Moreover, the number of essays specifically about Plato's language philosophy was limited about five essays, while the others wrote about Plato and other philosophers' language conceptions together and made some comparisons. 


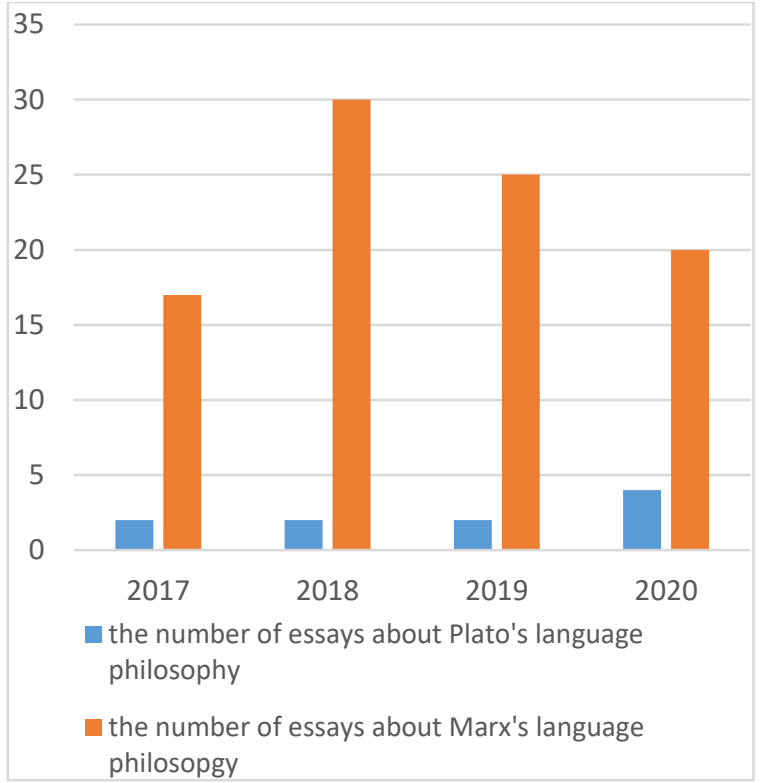

Figure 2. The comparison between the number of essays about Plato' s language philosophy and Marx' s language philosophy

Compared to the essays writing about Marx's language philosophy, the line graph implied that the number of essays about Marx was far higher than that about Plato in the latest five years and the year 2018 was the most obvious one. Although in 2021, the distance between them was smaller, it also had nine essays distance.

In conclusion, the research and learning of Plato's language philosophy were limited and not enough, especially for the specialized research of the language philosophy of Plato. Therefore, some solutions must be taken to inspire the exploration of Plato's language philosophy.

\section{THE SOLUTIONS OF DEVELOPING PLATO'S LANGUAGE PHILOSOPHY IN CHINA}

Because of the less knowledge of Plato's language philosophy, some solutions will solve this problem. As Plato's era was different from new China and his fundamental conceptions of ancient Greek were not close to traditional Chinese philosophy ideas, the central points of the solutions are deleting western abstract philosophy conceptions, which were not fitting into Chinese minds and adding the ideas of traditional Chinese philosophers', such as, Zhuangzi and Laozi. In order to realize the era value of Plato's language philosophy, some points of Marx should be inserted, as it is the official philosophy in new China. Then, Plato's language philosophy can also interpret and integrate into Confucius' theory of name correction.

\subsection{The Integration with Traditional Chinese Philosophy Conceptions}

\subsubsection{The Absorption of Conventional Chinese Language Philosophy}

Some traditional western logic, which was abstract, rational, and speculative, should be lessened in order for the Chinese to accept Plato's language philosophy, as these properties were not suitable for Chinese philosophy conceptions: feeling and intuition. Instead, Plato's language philosophy should be localized and draw lessons from some Chinese philosophers' ideas.

For instance, Plato took position of speech-centrism, which meant the first place was the oral speech and the second place writing speech existed when the oral speech was absent and it was the distortion of oral speech. The invention of writing words was harmful to people's memories, as before people's birth, they had owned the truth of the world, but once becoming human beings, they forgot about that. Therefore, to gain the truth, people should memorize it, writing words hindered the memories and blocked the way of finding the truth.[7] Furthermore, Zhuangzi thought the whole language system could not express the truth and essential of Tao, as his opinion was Tao noumenon. However, he believed the speech theory, which meant language was just the tool of expressing the emotions and it was not the ultimate aim, but the understanding Tao was, for instance, not saying what you mean and forgetting what you mean.[7] This silent and feeling theory of language fit into Chinese language and literature minds and it was easy to be accepted by Chinese and Plato's language philosophy can absorb some of this. Also, Plato's negative attitude toward writing words may harm the spreading of knowledge and emotion. Learning the speech theory can give the writing language some practical usages to explore the world.

Another example is the philosophy of Zhuangzi just implied the poetic properties, such as mental fasting, sitting, and heaven and earth coexist with ego.[7] Moreover, Laozi thought language could not clearly express the world and his ideas were realistic and pure. Therefore, Laozi disagreed with factitious decorations of language, and even language would destroy the truth of Tao. Also, he utilized nothing to express the statement of the beauty of speechless.[8] This case implied that the Chinese accept natural and intuitive language rather than logical and rational reasoning. So, to integrate into traditional Chinese minds, Plato's language philosophy can also adapt to some of the poetic properties and naturalism and dislodge some rational elements, which are difficult for the Chinese to understand. 


\subsubsection{The Insertion of Plato' s Language Philosophy into Chinese Language Philosophy}

In China, when professionally studying philosophy, learners should first learn Chinese philosophy and ignore western philosophy. However, many Chinese philosophical problems have lasted for thousands of years, and sometimes, using the conceptions of western philosophy can provide another thought to solve that. In this way, the Chinese can realize the importance of western philosophy and the connection between Chinese philosophy and western philosophy, which is a good way for spreading western philosophy and integrating it into Chinese philosophy.

An example is Hu Shi, who explored the origin of name correction and thought names were an image and image was related to Plato's ideal and form, in which way, Plato's language philosophy was transferred to traditional Chinese philosophy. Moreover, Feng Youlan referred to the name as an object's element or definition and interpreted the name correction as it came closer to its ideal or definition[11]. Also, it is commonly known that Plato's ideal is boundary and accurate and is also the basis of the real world. Using this theory, Confucius's name correction theory can also be explained as the name is clear and accurate and has a decisive impact on reality[11].

Using Plato's language philosophy to explain the problem of Chinese philosophy can let the Chinese understand their philosophy and know the benefits of Plato's language philosophy. More importantly, using the comparison method when learning Chinese philosophy can also help spread Plato's language philosophy.

\subsection{The Absorption of New-age Marxism Language Philosophy}

In China, Marxism philosophy is the official philosophy and it represents the development of advanced philosophy and the basic code of thought[9]. Therefore, Plato's language philosophy should coincide with the basic principles of Marxism and learn from the strengths of it, only in this way can the official support Plato's language philosophy to develop in China, Chinese people accept it and help develop the advanced culture in China.

For example, Plato's theory of the origin of language was Recsaid, which meant language should be in the ideal world and only outstanding soul could see it, and this point was mysticism and agnosticism[10]. However, Marx thought the emergence of language resulted from communications with others, and that language was the relationship between people and the environment[10]. In this case, Plato's language point not has the social and practical view and cannot develop China's society in a real and practical way. Therefore, it can absorb some points of Marxism language conceptions, encouraging people to socialize and form an interrelated philosophy culture. Moreover, agnosticism is thought wrong in China and does not coincide with the Chinese basic philosophy view.

Another example is, as mentioned above, Plato's language philosophy aimed to understand the world's truth. However, Marx thought the language was realistic, practical, scientific, and revolutionary, and the goal was to reconstruct the world[9]. So, the main aim of language philosophy of Plato is not to make numerous contributions to society, and it also can learn about the opinion of practical and revolutionary of Marxism. In this way, it can benefit for China's society by constantly redeveloping and reconstructing the society.

\section{CONCLUSION}

In summary, this essay introduces basic knowledge of Plato's language philosophy: the reference of names, the nature of the statement, and language and reality. Then, this essay provides a graph illustrating the limited amount of papers writing about Plato's language philosophy. Therefore, some solutions will be proposed. Firstly, some traditional and new-age Chinese philosophy elements should be added into Plato's language philosophy, such as Taoists and Marx. Secondly, some properties of Plato's language philosophy should be inserted into traditional Chinese philosophy for interpreting it and giving it more meaning. In these two ways, Plato's language philosophy can be helped to spread in China.

However, these solutions just remain at the level of theory. For really practicing them, these solutions should be modified and improved in the real world and fit into the situation of China. Also, it can refer to the experience of Buddhism absorbing the conceptions of Taoists and Taoism in order to spread in China.

Therefore, if Plato's language philosophy will be widespread in China, it can enrich the meaning of Plato's language philosophy and even Chinese native philosophy and provide more chances for people to learn about philosophy deeply and result in a situation in which hundred flowers are competing in the philosophical circle.

\section{REFERENCES}

[1] Zhang wave.From "language" to "real" - new exploration into Plato language philosophy.Modern philosophy, 2020 (1): 79-88.

[2] Wang Xiaochao, Introduction: Study Plato in China. Special Theme, 2013(4):527-529. 
[3] (Ancient Greece) The Complete Works of Plato: Volume 2 [M]. Wang Xiaoqiu Translation Beijing: People's Publishing House, 2003.

[4] (Ancient Greece) The Complete Works of Plato: Volume 3 [M]. Wang Xiaoqiu Translation Beijing: People's Publishing House, 2003.

[5] (Ancient Greece) The Complete Works of Plato Plato: Volume 4 [M]. Wang Xiaoqiu Translation Beijing: People's Publishing House, 2003.

[6] Chen Yuanyuan. Plato Language Philosophy. Huazhong University of Science and Technology, 2006.

[7] Wang Yanhua, Du Heng. Plato compares with the philosophical view of Zhuangzi language [J]. Literature Education, 2015,000(006):P.44-45.

[8] Jiang Hong. Language exploration from Plato's philosophical system [J]. Journal of Shandong Vocational and Technical College of Business, 2008,8 (006): 73-76.

[9] sings. From the reality of Plato "King of Philosophy" [J]. Journal of Hubei College for Nationalities (Philosophy and Social Sciences), 2002 (05): 98-99.

[10] Liu Si Kum. Philosophical Thoughts on the Origin of Language: From Plato to Marx [J]. Journal of Suzhou Institute of Science and Technology: Social Sciences, 2014 (3): 1-7.

[11] Gou Dongfeng. Platonism - - in modern Chinese philosophy is centered on the study of Confucius [J]. Wuling Journal, 2012,000 (003): 53-58. 